\title{
Early Event-related Potential Effects of Syllabic Processing during Visual Word Recognition
}

\author{
Manuel Carreiras ${ }^{1}$, Marta Vergara' ${ }^{1}$, and Horacio Barber ${ }^{2}$
}

\begin{abstract}
A number of behavioral studies have suggested that syllables might play an important role in visual word recognition in some languages. We report two event-related potential (ERP) experiments using a new paradigm showing that syllabic units modulate early ERP components. In Experiment 1, words and pseudowords were presented visually and colored so that there was a match or a mismatch between the syllable boundaries and the color boundaries. The results showed color-syllable congruency effects in the time window of the P200. Lexicality modulated the N400 amplitude, but
\end{abstract}

\section{INTRODUCTION}

Reading is a very important cognitive ability, and how readers extract the meaning of a word is a central question in understanding visual word recognition. It has been shown that sublexical representations could mediate between a word's constituent letters and the lexical level, suggesting that readers do not identify bisyllabic words as a whole (e.g., Carreiras \& Perea, 2002). However, although much progress has been made on how readers decode monosyllabic words, processing of multisyllabic words has been largely ignored, despite the prevalence of these in many languages. Most current computational models of visual word recognition assume that abstract letter representations are directly connected to word form representations, underestimating the possible role of sublexical units such as syllables (e.g., Coltheart, Rastle, Perry, Langdon, \& Ziegler, 2001; Grainger \& Jacobs, 1996; Seidenberg \& McClelland, 1989; McClelland \& Rumelhart, 1981; see, however, Ans, Carbonell, \& Valdois, 1998; Plaut, McClelland, Seidenberg, \& Patterson, 1996). Indeed, for the sake of simplicity, most visual wordrecognition models are essentially designed for short and monosyllabic words (but see Ans et al., 1998). Obviously, it is easier to work with monosyllabic words, especially when the main concern is the nature of representational structures and the organization of

\footnotetext{
${ }^{1}$ Universidad de La Laguna, Spain, ${ }^{2}$ University of California, San Diego
}

no effects of this variable were obtained at the P200 window. In Experiment 2, high- and low-frequency words and pseudowords were presented in the congruent and incongruent conditions. The results again showed congruency effects at the P200 for low-frequency words and pseudowords, but not for high-frequency words. Lexicality and lexical frequency effects showed up at the N400 component. The results suggest a dissociation between syllabic and lexical effects with important consequences for models of visual word recognition. the mental lexicon. Despite early attempts to find a perceptual unit involved in the recognition of polysyllabic words (Taft, 1979; Taft \& Forster, 1976) or the suggestion that sublexical input phonology could be coded syllabically in an interactive activation model (Ferrand, Segui, \& Grainger, 1996), there is no current computational model that can handle polysyllabic words. Thus, we are in need not only of computational models that can also account for multisyllabic words (see Carreiras \& Grainger, 2004) but also of more empirical evidence to help constrain such models.

A few previous studies have analyzed the impact of lexical processing on electrophysiological measures. Modulations of $\mathrm{N} 400$ amplitude, a negative deflection peaking around $400 \mathrm{msec}$ after word presentation, have been reported after manipulation of lexical variables. For example, N400 amplitude is modulated by the orthographic neighborhood size of words presented in word lists, which could indicate that this component is sensitive to the level of lexical activation (Holcomb, Grainger, \& O'Rourke, 2002). Similarly, N400 amplitude varies as a function of lexical frequency, being larger for low-frequency words than for high-frequency words (e.g., Neville, Mills, \& Lawson, 1992; Bentin, McCarthy, \& Wood, 1985). However, this component is also sensitive to contextual constraints (Kutas \& Federmeier, 2000), and lexical frequency interacts with word position in sentences, being entirely absent for sentence-final words (Van Petten \& Kutas, 1990). Thus, it seems that the N400 is related to lexical access and semantic integration processes. Other reports (Sereno \& Rayner, 2003; 
Sereno, Rayner, \& Posner, 1998) have claimed effects of "wordness" (words vs. nonwords) and word frequency (high- vs. low-frequency words) on event-related potential (ERP) components that occur considerably earlier than the N400, that is, the P100 and N150 (and see also ter Keurs, Brown, \& Hagoort, 2002; Münte et al., 2001; ter Keurs, Brown, Hagoort, \& Stegeman, 1999; King \& Kutas, 1998; Osterhout, Bersick, \& McKinnon, 1997, for early ERP effects of word class and word frequency).

Electrophysiological evidence with regard to the sublexical features of words in the visual domain is sparse. Rugg (1984a, 1984b) obtained a difference in a late negative component (N450) when subjects were asked to discriminate rhyming from nonrhyming pairs of letter strings. He attributed this effect to phonological incongruity because the effect was the same for words and nonwords. Bentin, Mouchetant-Rostaing, Giard, Echallier, and Pernier (1999) found one negative potential peaking at around $170 \mathrm{msec}$ (N170) when comparing orthographic and nonorthographic stimuli, a potential peaking at around $320 \mathrm{msec}$ (N320) when comparing pronounceable and nonpronounceable letter strings in the rhyme detection task, and a negativity peaking at around $350 \mathrm{msec}$ (N350) when comparing phonologically legal and phonologically illegal orthographic patterns in a lexical decision task (see also Schendan, Ganis, \& Kutas, 1998, for similar comparisons between different types of linguistic and nonlinguistic stimuli).

To date, most ERP studies have reported effects of both lexical and sublexical variables on rather late components. The lack of earlier effects associated to sublexical representations in previous studies could be due to the tasks, stimuli, languages used, or to other unknown factors. For instance, the use of some tasks (e.g., rhyming tasks) may tap postlexical processes instead of prelexical phonological processes, and the use of short words in languages with an opaque orthography such as English may induce readers to rely more on a direct lexical route. Thus, cross-linguistic comparisons using adequate tasks are essential to determine the commonalities and differences of word processing in the brain.

\section{Syllables in Visual Word Recognition}

Behavioral studies have highlighted the importance of syllables as sublexical representations, especially by manipulating syllable frequency (see Álvarez, Carreiras, \& Taft, 2001; Perea \& Carreiras, 1998; Carreiras, Álvarez, \& de Vega, 1993, for evidence in Spanish; Conrad \& Jacobs, 2004, in German; and Mathey \& Zagar, 2002, in French). For example, Carreiras et al. (1993) found that Spanish words starting with high-frequency syllables were responded to more slowly than words starting with low-frequency syllables when they were visually presented in a word list and the participants asked to make a lexical decision task. Converging evidence of the use of the syllable as a sublexical unit in Spanish has also been obtained using masked primes that shared the initial syllable with the target word (Álvarez, Carreiras, \& Perea, 2004; Carreiras \& Perea, 2002). For instance, Carreiras and Perea (2002; Experiment 3) used monosyllabic (ZINC) and CV.CV disyllabic words (RA.NA) ${ }^{1}$ as targets. The results showed a significant syllabic priming effect for the disyllabic words (ra.jo-RA.NA relative to $c u$. $f_{O}-R A . N A$ ). In contrast, monosyllabic words were not affected by related primes that shared the first two letters with the target (ziel-ZINC vs. flur-ZINC). Likewise, Álvarez, Carreiras, and Perea (2004) found significant priming effects for disyllabic prime-target pairs that shared the first three letters and the first CV syllable (e.g., ju.nas-JU.NIO) relative to disyllabic pairs that shared the first three letters but not the first syllable (jun.tu-JU.NIO).

Using the ERP technique, Barber, Vergara, and Carreiras (2004) examined the effects of syllable and word frequency in lexical access in Spanish. They found that high-frequency words produced an amplitude reduction of the N400 component as compared with lowfrequency words, confirming previous reports in other languages. In contrast, the frequency of the first syllable of a word elicited a reverse pattern. Words starting with high-frequency syllables were associated with more negative amplitudes than words starting with low-frequency syllables in the N400 range. In addition, Barber et al. found that the onset of the syllable frequency effect was earlier (150 msec) than that of the word frequency effect (350 $\mathrm{msec}$ ), indicating that these two variables could start to influence the word recognition process at different moments.

These syllable frequency effects have been interpreted in terms of an early syllabic activation that fires lexical candidates with a common first syllable, and a later competition among these word units in an interactive activation model (see Grainger \& Jacobs, 1996). The basic assumption is that not only orthographic neighbors (i.e., words that share all letters but one, e.g., cosacasa-Spanish words for thing-bouse; see Andrews, 1997 , for a review on orthographic neighbors) are activated in the process of visual word recognition, as is usually assumed, but also syllabic neighbors, that is, words that share a syllable, especially the first one, such as cosa-codo-thing-elbow (see Taft \& Forster, 1976). Thus, the lexical unit corresponding to the high-frequency Spanish word cosa ("thing") would be partially activated (or accessible) when the lower frequency syllabic neighbor codo ("elbow") is presented.

Taken together, these findings suggest that visual word recognition might be structured syllabically, at least for languages with clear syllable boundaries. Spanish has very transparent orthography and the syllable boundaries are very clear, and thus it is a suitable language 
in which to look for possible early ERP evidence of sublexical representations.

The goal of the present experiments is to track the time course of the availability of syllable representations during visual word recognition. We presented Spanish words in two different colors such that the color boundary could match or mismatch the syllable boundaries. If participants in our experiments perceive letter strings that form words chunked into syllable units, that is, if they analyze written words into syllables, they should experience interference in the incongruent conditions (i.e., when the syllable boundary does not match the color boundaries) as compared with the congruent condition in which there is a match between the syllable boundaries and the color boundaries, or the baseline condition in which the word is all in one color.

The syllabic effects previously reported have been found both for high- and low-frequency words. The interaction with word frequency reached significance only on very rare occasions (e.g., Álvarez, Carreiras, \& Taft, 2001); nonetheless, the effects were always larger for low-frequency words. Dual-route models (e.g., Coltheart et al., 2001) assume that word reading involves a fast, direct pathway (lexical route) from orthography to the stored word forms and a slower, indirect pathway (nonlexical route) that involves a grapheme-phoneme transformation. Although the Coltheart et al. (2001) model applies only to monosyllabic words, it is important to examine whether ERPs reveal early differences in the sublexical processing of high- and low-frequency polysyllabic words. Such differences, if any, were not well captured by behavioral measures. Therefore, we will examined syllabic effects for low-frequency words and pseudowords in Experiment 1 and for high- and low-frequency words as well as for pseudowords in Experiment 2.

\section{EXPERIMENT 1}

Colored words and pseudowords were presented for a lexical decision task. Stimuli were presented in two colors (red and green) such that in the congruent condition the first syllable was in one color and the second syllable in the other color (i.e., the color matched the syllable boundary), whereas in the incongruent condition, color and syllable boundaries did not match. In the baseline condition, the entire stimulus was presented in only one color. To the extent that syllabic processing is an important early step in visual word recognition, the incongruent condition should interfere with stimulus recognition compared with the congruent and baseline conditions, which might influence early electrophysiological responses. In addition, we expect to obtain the classic lexicality effect influencing the N400 previously reported in the literature. More importantly, however, we should observe a dissociation between the electrophysiological responses of color- syllable congruency and lexicality if they are tapping two different cognitive processes or two different moments of the same process during the time course of visual word recognition.

\section{Results}

\section{Behavioral Measures}

First, reaction times and error rates were analyzed. Incorrect responses (5\%) were excluded from the latency analysis. In addition, to avoid the influence of outliers, reaction times less than $300 \mathrm{msec}$ or greater than 1500 msec (less than $0.6 \%$ of the data) were excluded. The percentage of trials that were removed due to incorrect responses and the screening procedure was similar in all the conditions.

Analyses of variance (ANOVAs) based on the participant and item response latencies and percentage of errors were conducted based on a 3 (congruency: baseline, congruent, incongruent) $\times 2$ (lexicality: words, pseudowords) design. The mean lexical decision time and the percentage of errors on the words in each experimental condition are displayed in Table $1 .^{2}$

The ANOVA on the latency data showed an effect of lexicality: $F 1(1,23)=63.1, p<.001 ; F 2(1,235)=113$, $p<.01$. On average, words were responded to more rapidly than pseudowords. The ANOVA on the error rates showed the same lexicality effect: $F 1(1,23)=25.6$, $p<.001 ; F 2(1,235)=5.41, p<.05$. Words were responded to with a lower percentage of errors than pseudowords. No other effects were significant.

\section{Electrophysiological Measures}

The ERP grand averages of two representative electrodes, corresponding to the $\mathrm{F} 3$ and $\mathrm{P} 3$ positions of the 10/20 system, selected according to the topographical distributions of the effects, and time-locked to the onset of the stimuli are shown in Figure 1A for the three congruency conditions (baseline, congruent, and incongruent) as well as for words and pseudowords. Figure 1A reveals differences between the congruency conditions coinciding in latency with the P200 component (between 180 and $260 \mathrm{msec}$ ), with the amplitude values of the incongruent condition more positive

Table 1. Mean Lexical Decision Times (Milliseconds) and Percentage of Errors (in Parentheses) on Words and Pseudowords in Experiment 1

\begin{tabular}{lllc}
\hline & \multicolumn{3}{c}{ Congruency Syllable-Color } \\
\cline { 2 - 4 } & Baseline & Congruent & Incongruent \\
\hline Words & $694(7.5)$ & $699(7)$ & $713(5.6)$ \\
Pseudowords & $774(3)$ & $776(2.2)$ & $773(3.1)$ \\
\hline
\end{tabular}




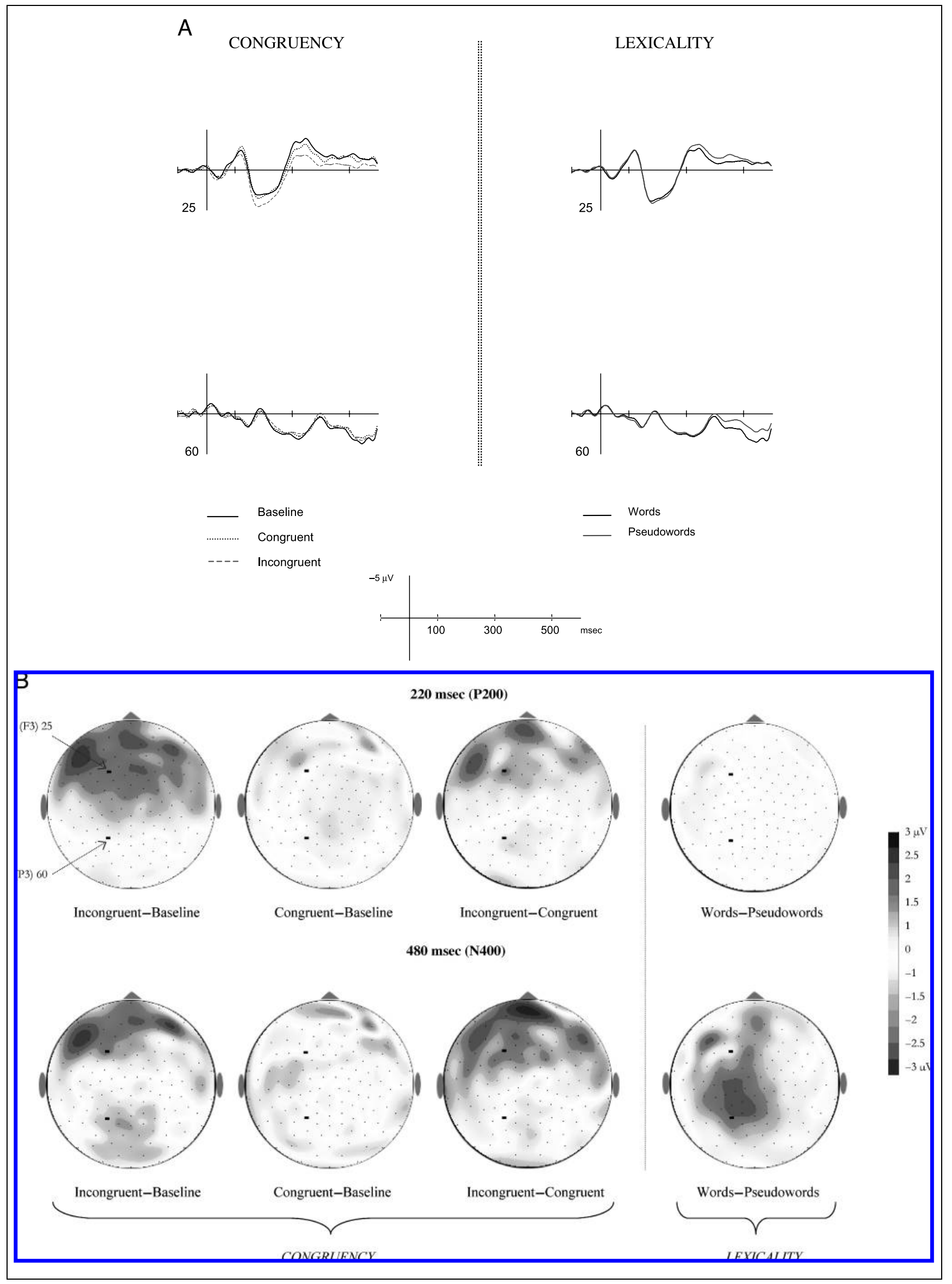


than those of the baseline and the congruent condition. No differences corresponding to lexicality were found prior to $300 \mathrm{msec}$. However, a clear effect of lexicality appears in a later window (350-500 msec), with pseudoword ERPs more negative than word ERPs. Topographical voltage maps for the comparisons among the three congruency conditions and for the words versus pseudowords contrast (when collapsed over the three congruency conditions) are represented in Figure 1B. Statistical analysis supports these observations.

\section{P200 Segment}

The ANOVA with mean amplitude values of the 180- to 260-msec time epoch, including the factors congruency (baseline, congruent, and incongruent), electrode regions (anterior, central, and posterior), hemisphere (right vs. left), and lexicality (words vs. pseudowords), did not show any effect of lexicality $(F<1)$, but showed a significant interaction between the factors congruency and electrode, $F(4,92)=3.4, p<.05, \varepsilon=61$, indicating that in the anterior region the incongruent condition was more positive-going than the baseline, $F(1,23)=$ $4.6, p<.05$, and marginally more significant than the congruent condition, $F(1,23)=3.02, p=.09$, but the baseline and the congruent conditions did not differ $(F<1)$. The interaction between the factors lexicality and congruency was not significant $(F<1)$. No other effects were reliable.

\section{N400 Segment}

The ANOVA with the average values of the 350- to 500-msec time epoch, including the factors congruency (baseline, congruent, and incongruent), electrode regions (anterior, central, and posterior), hemisphere (right vs. left), and lexicality (words vs. pseudowords), showed a main effect of lexicality, $F(1,23)=12.9$; $p<.005$, as well as an interaction of Lexicality $\times$ Electrodes, $F(2,46)=10 ; p<.001, \varepsilon=0.72$, which was maintained after the data normalization, $F(2,46)=$ 8.3; $p<.005, \varepsilon=0.71$. Effects of lexicality (nonwords showing a larger negative amplitude than words) were obtained in the anterior, $F(1,23)=24.8 ; p<.001$, and in the central, $F(1,23)=19.6 ; p<.001$, but not in the posterior area $(F<1)$. In addition, there was an interaction between congruency and electrodes, $F(4,92)=$ $4.7 ; p<.05, \varepsilon=0.63$, due to a larger negative amplitude for the baseline than the incongruent condition in the anterior area, $F(1,23)=6.3 ; p<.05$. The interaction between the factors lexicality and con- gruency was not significant $(F<1)$. No other effects were reliable.

As can be seen in the averaged waveforms (Figure 1A), the P200 amplitude modulations are followed by a modulation of the N400. In this case, the analysis of the N400 is complicated by the preceding differences in P200 amplitudes. One way to compensate for this baseline problem is to renormalize the waveforms with respect to the P200 latency window (Hagoort, 2003). The omnibus ANOVA on the renormalized amplitude values in the 350- to 500-msec latency range showed again a main effect of lexicality, $F(1,23)=33.3 ; p<.005$, as well as an interaction of Lexicality $\times$ Electrodes, $F(2,46)=9.1 ; p<.001, \varepsilon=0.69$. Effects of lexicality (nonwords showing a larger negative amplitude than words) were obtained in the anterior, $F(1,23)=39.9$; $p<.001$, and in the central, $F(1,23)=21.1 ; p<.001$, but not in the posterior area, $F(1,23)=2.3$. However, neither the main effect of congruency $(F<1)$ nor the interaction between congruency and electrodes was significant $(F<1)$.

\section{Discussion}

The results of Experiment 1 showed no color-syllable congruency, but a lexicality effect in the behavioral measures. In addition, they showed a temporal and spatial dissociation of color-syllable congruency and lexicality effects in the ERPs. Color-syllable congruency effects occurred as early as $180 \mathrm{msec}$ and had a frontal distribution, whereas lexicality effects commenced at around $300 \mathrm{msec}$ and had a more central distribution. There was an amplitude increase for the color-syllable incongruency condition as compared with the colorsyllable congruency and the baseline conditions in the P200 time window. In addition, pseudowords showed larger N400 amplitudes than words. The onset difference between color-syllable congruency and lexicality effects is in line with that reported in a previous experiment in which syllable frequency and word frequency were manipulated (Barber et al., 2004) and suggests that these effects could be associated with different word recognition processes.

The color-syllable congruency effect could be related to interference of the syllabic/phonological processing of the stimuli and the activation of lexical candidates. This early effect is in line with the above-mentioned proposal (e.g., Carreiras \& Perea, 2002; Carreiras et al., 1993) that syllables trigger the activation of lexical candidates during the analysis of orthographic or phonological representations. The color-congruent and the

Figure 1. (A) ERP waves corresponding to the three congruency conditions and to the words and pseudowords collapsed across the three congruency conditions in two representative electrodes (25 and 60) that correspond with the F3 and P3, respectively, in the 10/20 system. (B) Topographical distribution of the three congruency comparisons and of the word versus pseudoword contrast at 220 and at 480 msec. The analyzed windows are indicated with two lines (first window, 180-260 msec; second window, 350-500 msec). Negative amplitude is plotted upward. 
baseline conditions would trigger the activation of lexical candidates that share the first syllable with the target item. Most of these activated candidates would also be inhibited, via lateral inhibition, in order to identify the correct lexical item. These activation processes presumably are interfered with when the words display a mismatch between color and syllable boundaries, resulting in a lower number of activated candidates or in a lower activation of these candidates. In this way, ERP amplitude differences in this experiment could be related to differences in the lexical activation triggered by syllables. This explanation assumes that words are decomposed into syllables during the recognition process. However, this is possibly not the case for high-frequency words, which could be recognized faster by means of a direct route without syllabic decomposition. This possibility was addressed in Experiment 2 .

\section{EXPERIMENT 2}

The results obtained in Experiment 1 showed a syllablecolor congruency effect at the P200 time window, and this effect occurred both for words and for pseudowords. As words in Experiment 1 were of low lexical frequency, Experiment 2 was designed to address whether the syllabic effects also occur for high-frequency words. In addition, in Experiment 1, although the baseline and the congruent conditions showed similar ERPs, statistical differences were obtained only between the incongruent condition and the baseline but not between the congruent and the incongruent conditions. Therefore, in order to gain more statistical power, the three congruency conditions were reduced to two. The congruent and the incongruent conditions were used in Experiment 2 because the stimuli of these two conditions were physically more similar, as they were presented in two colors, whereas only one color was presented in the baseline condition.

\section{Results}

\section{Behavioral Measures}

Incorrect responses (3.64\%) were excluded from the latency analysis. In addition, to avoid the influence of outliers, reaction times less than $300 \mathrm{msec}$ or greater than 1500 msec (less than $0.5 \%$ of the data) were excluded. The percentage of trials that were removed due to incorrect responses and the screening procedure was similar in all the conditions.

ANOVAs based on participant and item response latencies and percentage of errors were conducted based on a 2 (congruency: congruent, incongruent) $\times$ 2 (lexicality: words vs. nonwords) design. The mean lexical decision time and the percentage of errors on the words in each experimental condition are displayed in Table $2 .^{3}$
Table 2. Mean Lexical Decision Times (Milliseconds) and Percentage of Errors (in Parentheses) on Words and Pseudowords in Experiment 2

\begin{tabular}{llc}
\hline & \multicolumn{2}{c}{ Congruency Syllable-Color } \\
\cline { 2 - 3 } & Congruent & Incongruent \\
\hline High-frequency words & $598(1)$ & $595(1.5)$ \\
Low-frequency words & $714(7.2)$ & $699(7.9)$ \\
Pseudowords & $792(4)$ & $723(3.6)$ \\
\hline
\end{tabular}

The ANOVA on the latency data showed an effect of lexicality: $F 1(1,28)=101.9, p<.001 ; F 2(1,314)=173$, $p<.001$. On average, words were responded to more rapidly than pseudowords. The congruency effect was also reliable: $F 1(1,28)=28.9, p<.001 ; F 2(1,314)=$ 94.3, $p<.001$. However, both main effects were qualified by an interaction between lexicality and congruency: $F 1(1,28)=22.1, p<.001 ; F 2(1,314)=54.6, p<.001$. The congruency effect was only reliable for pseudowords: $F 1(1,28)=36.5, p<.001 ; F 2(1,157)=118$, $p<.001$, indicating that pseudowords in the congruent condition were responded to more slowly than in the incongruent condition. The analyses of the error rates did not show any reliable effects.

Additional analyses only for words were performed, replacing lexicality by lexical frequency as a factor. ANOVAs based on participant and item response latencies and percentage of errors were conducted based on a 2 (congruency: congruent, incongruent) $\times 2$ (lexical frequency: high vs. low) design.

The ANOVA on the latency data showed an effect of frequency: $F 1(1,28)=160, p<.001 ; F 2(1,156)=181$, $p<.001$. On average, high-frequency words were responded to more rapidly than low-frequency words. The ANOVA on the error rates showed the same frequency effect: $F 1(1,28)=73.7, p<.001 ; F 2(1,156)=26.8$, $p<.001$. High-frequency words were responded to with a lower percentage of errors than low-frequency words. No other effects were reliable.

\section{Electrophysiological Measures}

The ERP grand averages, time-locked to the onset of the words and pseudowords, are represented in Figures 2 and 3 over six recording sites. Figure 2 shows the congruent and incongruent conditions for words (collapsed across high- and low-frequency words) and pseudowords, and Figure 3 shows the congruent and incongruent conditions for high- and low-frequency words. Visual inspection reveals clear differences in an early component (P200) in the responses to the congruent conditions as compared with the incongruent conditions for words and pseudowords (Figure 2), 
Figure 2. ERPs to the target words and pseudowords in the baseline, congruent, and incongruent conditions. Three left and three right hemisphere electrode sites are shown.

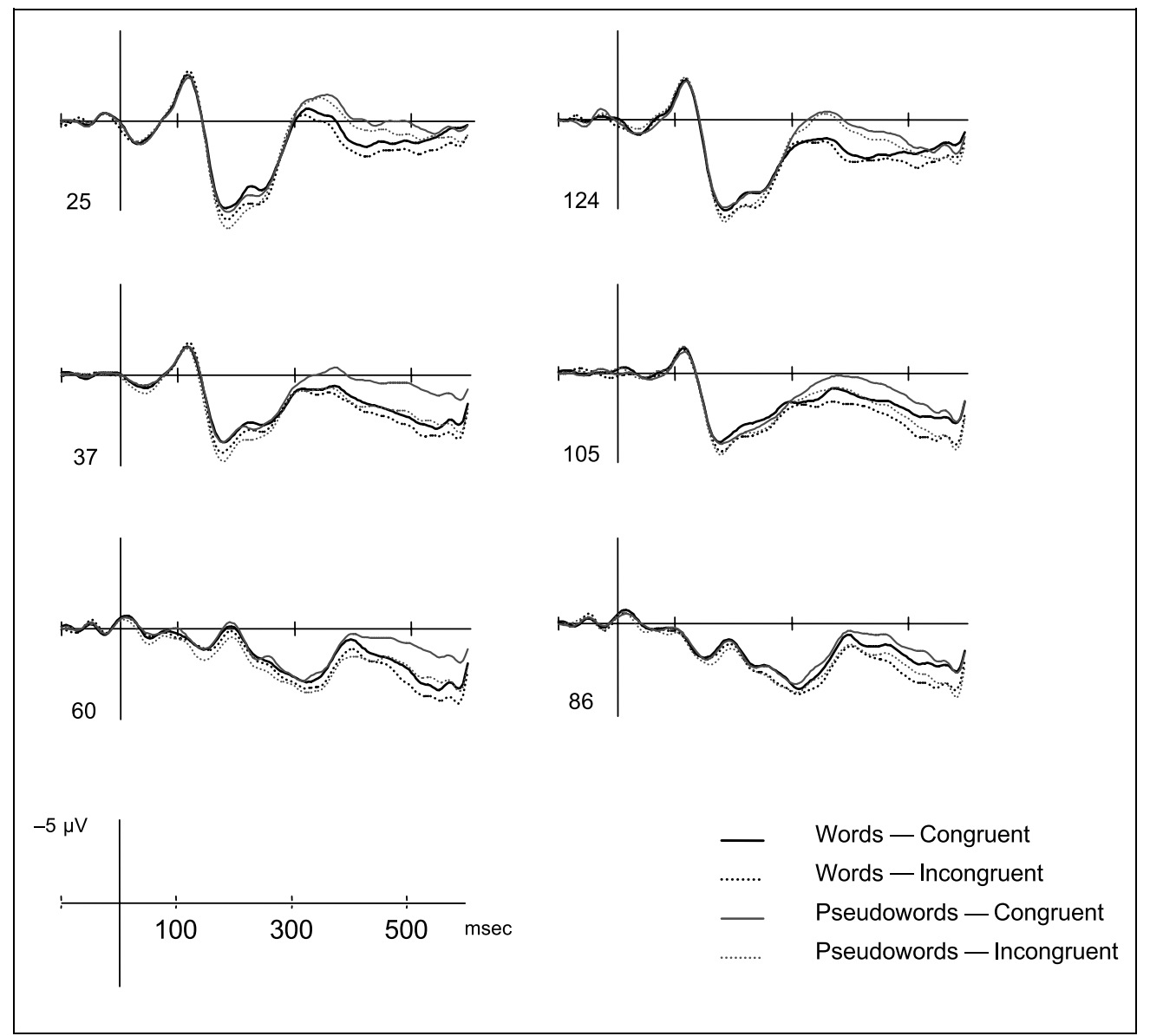

as well as for low-frequency words but not for highfrequency words (Figure 3). These differences are observed within the $180-$ to $260-\mathrm{msec}$ window. No differences corresponding to lexicality or lexical frequency appear in this time window. Furthermore, in a later window (350-500 $\mathrm{msec})$, effects of congruency again appeared for pseudowords and for low-frequency words but not for high-frequency words. In addition, the typical lexicality and lexical frequency effects are clearly seen; pseudoword ERPs were more negative-going than word ERPs and low-frequency word waves were more negative-going than high-frequency word waves. Figure 4 shows the topographical distribution over the scalp of all the above-described effects at the 180- to 260-msec and at the 350-500 msec windows. Statistical analysis supports these observations.

\section{P200 Segment}

The ANOVA with the average values of the 180- to 260-msec time epoch, including the factors congruency (congruent vs. incongruent), lexicality (words vs. pseudowords), electrode regions (anterior, central and posterior), and hemisphere (left vs. right), showed an interaction between congruency and electrode regions, $F(2,56)=6.96$, $p<.01, \varepsilon=0.60$. Congruency effects were found in the anterior, $F(1,28)=21.2, p<.001$, and the central, $F(1,28)=21, p<.001$, but not in the posterior region $(F<1)$, indicating that the incongruent conditions were more positive going than the congruent conditions. Lexicality effects were not found in this time window.

Additional analyses were performed for words only, including the factors (congruent vs. incongruent), lexical frequency (high vs. low), electrode regions (anterior, central, and posterior), and hemisphere (right vs. left), and showed an interaction between the factors congruency, lexical frequency and electrode regions, $F(2,56)=$ $6.85, p<.01, \varepsilon=0.71$, which was maintained (marginally significant) after data normalization, $F(2,56)=1.93$, $p=.16, \varepsilon=0.68$. No reliable effects were found for high-frequency words $\left(F_{S}<1\right)$. However, low-frequency words showed congruency effects, indicating that the low-frequency words in the incongruent condition were more positive-going than in the incongruent condition in the anterior, $F(1,28)=23.3, p<.0001$, and the central, $F(1,28)=16.6, p=.000$, but not in the posterior region $(F<1)$.

\section{N400 Segment}

The ANOVA with the average values of the 350- to 500-msec time epoch, including the factors congruency 
Figure 3. ERPs to the target high- and low-frequency words in the baseline, congruent, and incongruent conditions. Three left- and three righthemisphere electrode sites are shown.

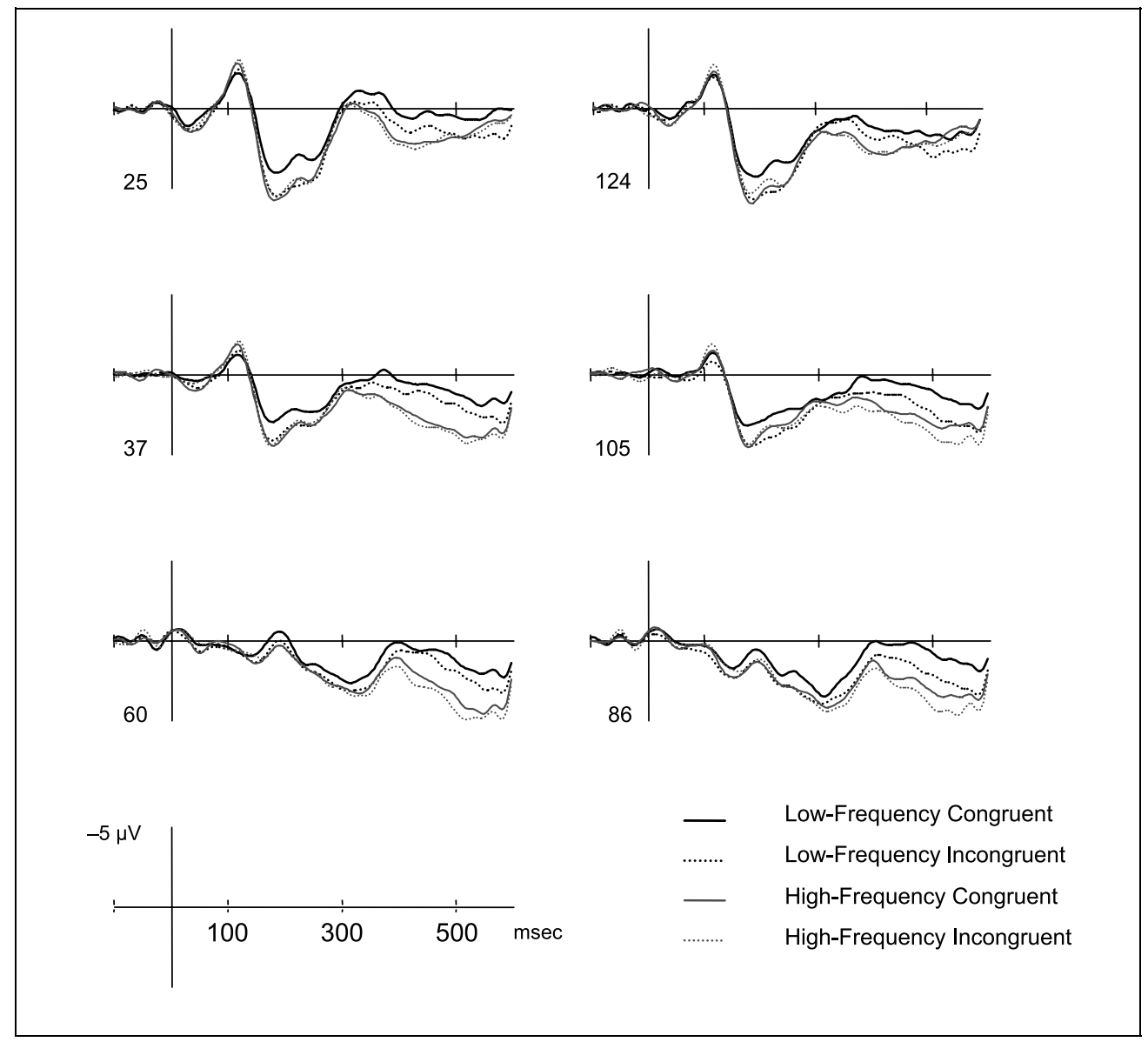

(congruent vs. incongruent), lexicality (words vs. pseudowords), electrode regions (anterior, central, and posterior), and hemisphere (left vs. right), showed a main effect of lexicality, $F(1,28)=22.07, p<.001$, which was qualified by an interaction with electrode regions, $F(2,56)=16.3, p<.01, \varepsilon=0.59$, which was maintained after data normalization, $F(2,56)=19.1 ; p<.001, \varepsilon=$ 0.61 . Lexicality effects indicating that nonwords were more negative-going than words were found in the anterior, $F(1,28)=37.2, p<.001$, and the central, $F(1,28)=13, p<.001$, but not in the posterior region $(F<1)$. Finally, there was also a main effect of congruency, $F(1,28)=23.3, p<.001$ : the congruent condition was more negative going than the incongruent condition.

Additional analyses for words only, including the factors congruency (congruent vs. incongruent), lexical frequency (high vs. low), electrode regions (anterior, central, and posterior), and hemisphere (right vs. left), showed a main effect of frequency [low-frequency words were more negative-going than high-frequency words, $F(1,28)=12.6, p<.005]$ and a main effect of congruency [the congruent condition was more negative going than the incongruent condition, $F(1,28)=6.53, p<.05]$. The interaction of congruency and lexical frequency was not reliable, $F(2,27)=1.5, p>$. . However, congruency effects were only reliable for the low-frequency words, $F(2,56)=5.1, p<.05$, but not for the high-frequency words, $F(2,56)=1.5, p>.1$.

Again, as can be seen in the averaged waveforms (Figures 2 and 3) the P200 amplitude modulations are followed by a modulation of the N400 time window. The omnibus ANOVA on the renormalized amplitude values in the 350- to 500-msec latency range for words and pseudowords showed a main effect of lexicality, $F(1,28)=38.9, p<.0001$, which was qualified by an interaction with electrode regions, $F(2,56)=28.6$, $p<.0001, \varepsilon=0.63$. Lexicality effects indicating that nonwords were more negative-going than words were found in the anterior, $F(1,28)=62, p<.001$, and the central, $F(1,28)=29.5, p<.001$, but not in the posterior region, $F(1,28)=1.62$. Finally, there was also a main effect of congruency, $F(1,28)=8.36, p<.01$ (the congruent condition was more negative going than the incongruent condition), as well as an interaction between congruency and electrodes, $F(2,56)=$ $5.41, p<.05$. Effects of congruency were found in the central, $F(1,28)=15.1, p<.001$, and the posterior, $F(1,28)=21.1, p<.001$, but not in the anterior region $(F<1)$.

Additional analyses for words only, including the factors congruency (congruent vs. incongruent), lexical 


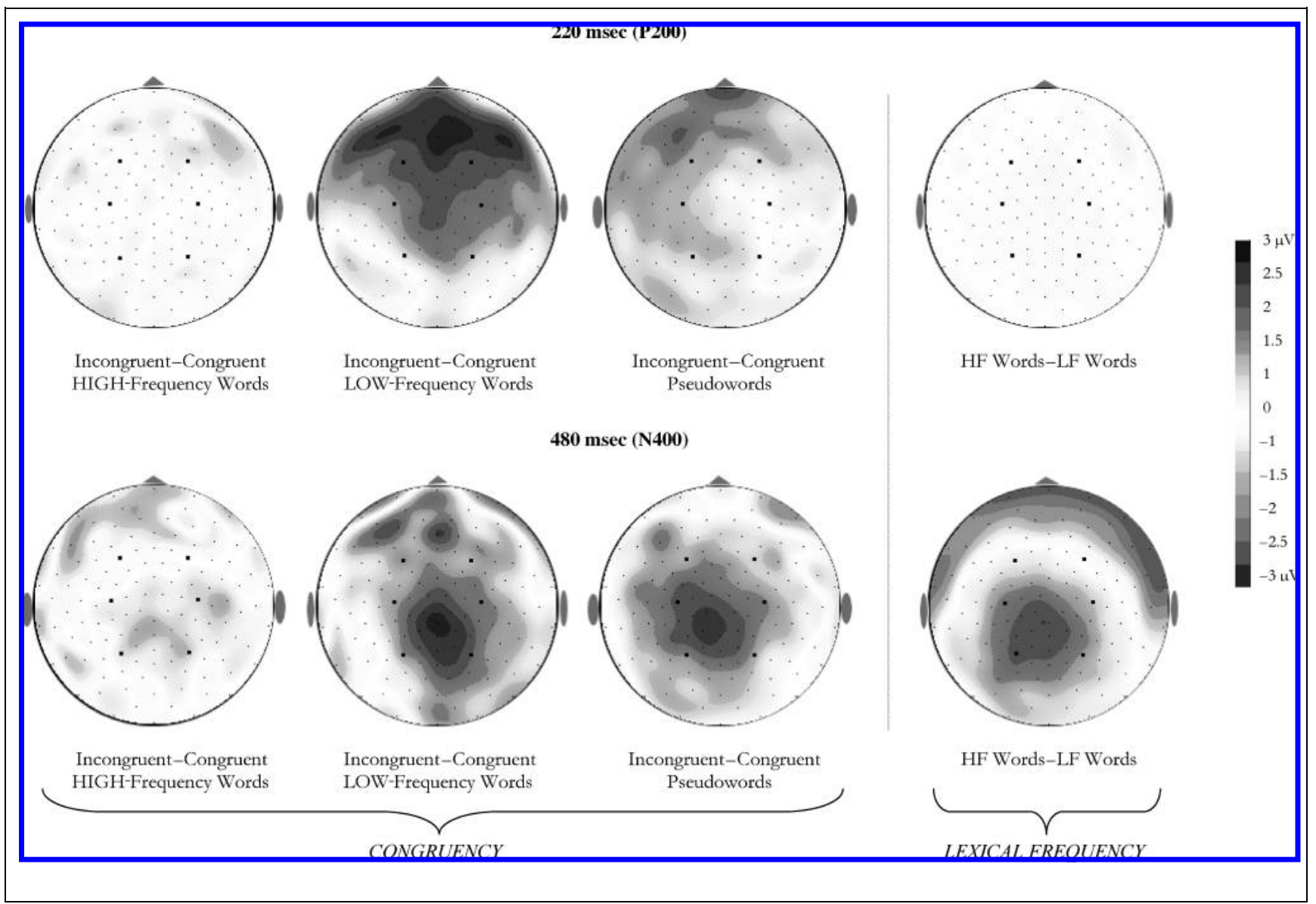

Figure 4. Topographical distribution of the congruency effect at $220 \mathrm{msec}$ and at $480 \mathrm{msec}$ in the high-frequency (HF) words, low-frequency (LF) words, and pseudowords.

frequency (high vs. low), electrode regions (anterior, central, and posterior), and hemisphere (right vs. left), showed a main effect of frequency [low-frequency words were more negative-going than high-frequency words, $F(1,28)=17.1, p<.001]$ as well as the interaction of lexical frequency and electrodes, $F(2,56)=$ $17.1, p<.001$. Effects of lexical frequency were more prominent in the central, $F(1,28)=31.9, p<.001$, and the posterior, $F(1,28)=13.5, p<.001$, than in the anterior region, $F(1,28)=4, p<.06$. Neither the effects of congruency, $F(1,28)=1.9$, nor the interaction of congruency and electrodes, $F(2,56)=1.9$, were significant.

\section{Discussion}

Effects of lexicality and lexical frequency were obtained in the behavioral measures. In addition, effects of colorsyllable congruency were found again in the P200 segment for pseudowords and for low-frequency words in the ERPs. No effects of congruency were found for highfrequency words. These early effects are consistent with the proposal that syllabic segmentation could only be occurring for low-frequency words and pseudowords but not for high-frequency words. We cannot discard, however, that high-frequency words are routinely syllabified, because our paradigm might not be sensitive enough to capture such a process. In fact, in previous studies, syllable frequency effects have been obtained for high-frequency words, although they were always much weaker than those obtained for low-frequency words, which suggests that syllables are involved in the processing of high-frequency words. Syllabic parsing may routinely occur for high-frequency words but may be quickly shadowed by the fast lexical access to the word itself.

Surprisingly, the amplitude of the response to highfrequency words in both congruency conditions was similar to that for low-frequency words in the incongruent condition. In any case, the fact that sublexical effects were not found for high-frequency words seems to suggest fast lexical access to these words via more weight of the direct lexical route without syllabic intermediation. In addition, the onset difference between the syllable congruency effect and the lexical frequency effect in this experiment is in line with that obtained in previous experiments wherein syllable and word frequency were manipulated (Barber et al., 2004) and 
suggests that these effects could be associated with different word recognition processes.

\section{GENERAL DISCUSSION}

The results of both experiments show that (1) colorsyllable congruency produced an early frontal ERP effect in the P200 time window. Color-syllable incongruent conditions produced more positive amplitudes at the P200 window than color-syllable congruent and baseline conditions. However, color-syllable congruency did not affect high-frequency words. (2) Lexicality (words vs. pseudowords) and lexical frequency effects (high- vs. low-frequency words) modulated N400 amplitudewords produced less negative amplitudes than pseudowords and high-frequency words produced less negative amplitudes than low-frequency words. (3) The time course and spatial distribution of the syllable-color congruency effects differ from those of lexicality and lexical frequency. Although the lexicality and lexical frequency effects started at $300 \mathrm{msec}$, the interaction between lexical frequency and congruency at the P200 time window suggests that lexical frequency affects the recognition process much earlier than this point: as early as $180 \mathrm{msec}$, when the color-syllabic effects started. In addition, the spatial distributions of colorsyllable congruency effects and lexicality or lexical frequency effects are different, which could indicate that these effects are associated with related but different processes.

Taken together, the present findings can be considered as further empirical support for the general notion that syllables are units of processing during visual word recognition (e.g., Álvarez, Carreiras, \& Perea, 2004; Barber et al., 2004; Carreiras \& Perea, 2002; Perea \& Carreiras, 1998; Carreiras et al., 1993). More importantly, they seem to suggest that syllables could be sublexical representations dissociable from lexical representations during the process of word recognition. The color-syllable congruency effect could be associated with some early activation of lexical candidates and a late interference that seems to happen in the case of low-frequency words and pseudowords. It has been proposed that syllables could play a role in the activation of lexical candidates (e.g., Carreiras \& Perea, 2002; Carreiras et al., 1993). In the present experiments, words with color-syllable congruency (or with just one color) trigger the proposed activation of lexical candidates that share the first syllable with the target item. Most of these activated candidates are then inhibited via lateral inhibition in order to identify the correct lexical item. This activation process presumably is interfered with when words display a color-syllable mismatching, resulting in a lower number of activated candidates or in a lower activation of these candidates and therefore in a lesser degree of lateral inhibition. In fact, the early congruency effects in the P200 window were followed not only by effects of lexicality and lexical frequency, but also of congruency in the N400 time window. The effects of congruency in the $\mathrm{N} 400$ window did not survive in Experiment 1 when waveforms were renormalized to compensate for the baseline problem in the P200 window. However, a main effect of congruency was still found in Experiment 2 after the baseline correction when words and pseudowords were analyzed together. Experiment 2 clearly shows that congruency effects are circumscribed to pseudowords and lowfrequency words. Color-syllable incongruent conditions seem to produce more positive amplitude at the P200 window and still less negative amplitudes than colorcongruent conditions at the N400 window. Indeed, the fact that late congruency effects were obtained in Experiment 2 but not in Experiment 1 may be due to the larger ratio of competition interference provoked by the congruent and incongruent conditions in Experiment 2 . The difference in syllable frequency between the congruent and incongruent conditions was larger in Experiment 2 than in Experiment 1 (see Table 3). It is important to note that this explanation not only fits with the present electrophysiological findings, but also with converging previous empirical evidence (e.g., Perea \& Carreiras, 1998; Carreiras et al., 1993), showing that whereas word frequency produces a facilitative effect in lexical decision tasks, syllable frequency produces an inhibitory effect (words with high-frequency syllables are

Table 3. Number of Nonexisting Syllables in Spanish and of Legal Syllables of the Spanish Language that Resulted in the Incongruent Condition, Type Frequency of these Legal Syllables, and Difference of Type Frequency between the Legal Syllables of the Incongruent Condition and the Type Frequency of the First Syllable of the Congruent Condition in Experiments 1 and 2

\begin{tabular}{|c|c|c|c|c|}
\hline & $\begin{array}{c}\text { Nonexisting } \\
\text { Syllables }\end{array}$ & $\begin{array}{c}\text { Legal } \\
\text { Syllables }\end{array}$ & $\begin{array}{c}\text { Type } \\
\text { Frequency } \\
\text { of Legal } \\
\text { Syllables }\end{array}$ & Difference \\
\hline \multicolumn{5}{|l|}{ Experiment 1} \\
\hline Words & 45 & 75 & 13.6 & 2.8 \\
\hline Pseudowords & 69 & 51 & 2.7 & 11.6 \\
\hline \multicolumn{5}{|l|}{ Experiment 2} \\
\hline HF Words & 40 & 40 & 15.8 & 33.5 \\
\hline LF Words & 38 & 42 & 18.2 & 15.3 \\
\hline Pseudowords & 116 & 44 & 14 & 25.2 \\
\hline
\end{tabular}

Note that the legal syllables can be isolated as such in Spanish, but such segmentation is not legally permitted in the context of each of the present stimuli (words and pseudowords). $\mathrm{HF}=$ high frequency; $\mathrm{LF}=$ low frequency. 
identified more slowly than those with low-frequency syllables).

The present findings pose important constraints for a new generation of computational models of visual word recognition. Despite the focus of current computational models on monosyllabic words, monosyllabic words are statistically much less common than polysyllabic words (except for short functional words), and thus it is important to develop a model of polysyllabic words. Ferrand et al. (1996) have proposed one such model, in which syllable-sized units were implemented both in sublexical input phonology and in sublexical output phonology. Sublexical input phonology is thought to be structured syllabically: Sublexical phonological codes (such as syllables) could receive activation from the letter level and send on activation to the word level, so that words that share one syllable can influence the process of word recognition (via lexical inhibition at the word level). This framework would capture why syllabic effects (syllabic frequency and syllabic congruency) are facilitative at an early moment because of the faster computation of units in the sublexical input phonology. However, later they become inhibitory because of the lateral inhibition mechanism. Syllabic neighbors may behave in a similar way to "orthographic" neighbors (e.g., $\cos a-c a s a$; thing-house): Syllabic neighbor words will compete in some way in the recognition process, and the degree of competition is a function of the relative frequency of the target word and its competitors (e.g., see Grainger \& Jacobs, 1996, for a computational model of visual word recognition and lexical decision). However, this has not yet been implemented in a computational model.

Finally, the present findings indicate that early ERP effects can be obtained for sublexical and lexical manipulations. A variety of sources of evidence suggests that lexical representations are available within around $250 \mathrm{msec}$ of word onset when measuring eye movements (Starr \& Rayner, 2001; Rayner, 1975, 1998). The present experiment showed an interaction between color-syllable congruency and lexical frequency between 180 and $260 \mathrm{msec}$ after stimulus onset. This finding suggests that syllabic representations are available very early, influencing the processing of pseudowords and low-frequency words. However, no effects of colorsyllable congruency were shown for high-frequency words, suggesting that the processor was sensitive to the frequency of words at this early moment. Therefore, the present effects are consistent with eye movement models in which lexical access is well under way within the first 200 msec of fixation.

In summary, in the present experiments the manipulation of sublexical information resulted in ERP effects at the P200 time window that were sensitive to the lexical frequency manipulation. The onset latency of these effects was earlier than that of lexical variables, which modulated the N400 component. To our knowledge, the present study and a recently published study by our group (Barber et al., 2004) are the first to report the modulation of ERP responses by sublexical representations at these early latencies.

\section{METHODS \\ Participants}

Twenty-eight (20 women) undergraduate students participated in Experiment 1 and 32 (22 women) undergraduate students took part in Experiment 2 in exchange for course credit. All of them were native Spanish speakers with no history of neurological or psychiatric impairment and with normal or correctedto-normal vision. Ages ranged from 19 to 28 years (mean, 21.1 years). All participants were right-handed, as assessed with an abridged Spanish version of the Edinburgh Handedness Inventory (Oldfield, 1971). Data of four participants from Experiment 1 and data from three participants from Experiment 2 were rejected before the analysis because of too many artifacts in the EEG record.

\section{Procedure}

Participants were seated comfortably in a darkened sound-attenuated chamber. All stimuli were presented on a high-resolution monitor that was positioned at eye level, $80-90 \mathrm{~cm}$ in front of the participant. The words were displayed in red and green lowercase Georgia 36 letters against a black background. The green color was defined in the RGB model according to the following values: red $=0$, green $=204$, blue $=0$. The red color was defined according to the following values: red $=$ 255 , green $=0$, and blue $=0$.

Participants performed a lexical decision task; that is, they were instructed to press one of two buttons on the response pad to indicate whether the letter string was a legitimate Spanish word or not. A response button was positioned beneath each thumb. For half of the participants, the right button was used to signal the "Yes" response and the left button was assigned the "NO" response. For the remaining participants the order was reversed.

Each trial began with a row of hash marks (\#\#\#\#\#\#) that appeared in the center of the screen and remained there for $500 \mathrm{msec}$. A blank screen interval of $150 \mathrm{msec}$ followed, and then the word was displayed for $225 \mathrm{msec}$. The trial ended with the participant's response or 2000 msec after the presentation of the word if the participant failed to respond. The intertrial interval varied randomly between 600 and $1300 \mathrm{msec}$ (see Figure 5). All items were presented in a different random order for each participant in four different blocks, with a break of 5 min between blocks in which the participant could rest and the impedances were checked. 


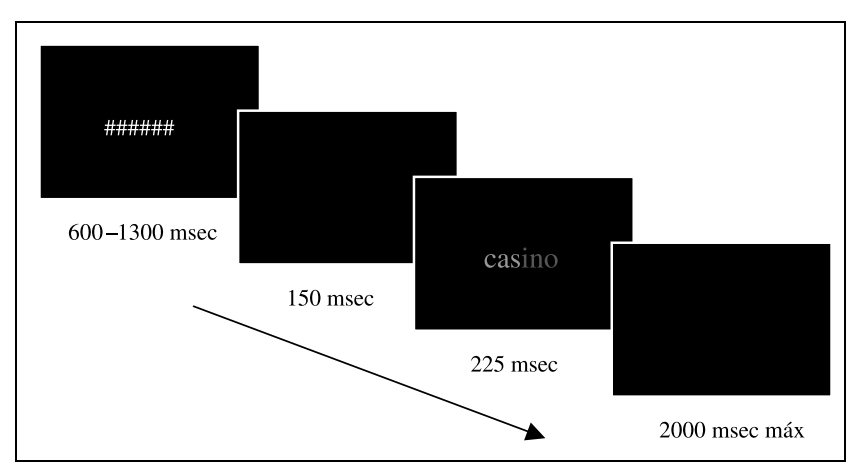

Figure 5. Sequence for each trial.

Fifteen warm-up trials were provided at the beginning of the session and were repeated if necessary. Participants were also asked to avoid eye movements and blinks during the interval when the row of hash marks was not present and they were directed to favor accuracy over speed in their responses. For this reason, reaction times were not analyzed. Each session lasted approximately $1 \mathrm{hr}$ and $15 \mathrm{~min}$.

\section{EEG Recording and Analyses}

Scalp voltages were collected from $\mathrm{Ag} / \mathrm{AgCl}$ electrodes using a 128-channel geodesic sensor net. Figure 6 shows the schematic distribution of the recording sites. The vertex electrode was used as reference, and the recording was re-referenced off-line to linked mastoids. Eye movements and blinks were monitored with supra- and infraorbital electrodes and with electrodes on the external canthi. Interelectrode impedances were kept below $30 \mathrm{k} \Omega$ (amplifier input impedance $>200 \mathrm{M} \Omega$ ). EEG was filtered with an analogue band-pass filter of $0.01-100 \mathrm{~Hz}$ (50-Hz notch filter) and a digital $35-\mathrm{Hz}$ low-pass filter was applied before analysis. The signals were sampled continuously throughout the experiment with a sampling rate of $250 \mathrm{~Hz}$.

Epochs of the EEG corresponding to $600 \mathrm{msec}$ after word onset presentation were averaged and analyzed.
Figure 6. Schematic flat representation of the 129 electrode positions from which EEG activity was recorded (front of head is at top). Channel nomenclature is by number. Approximate international 10/20 system localizations are marked. The colored electrodes are those grouped and analyzed in the six critical regions.

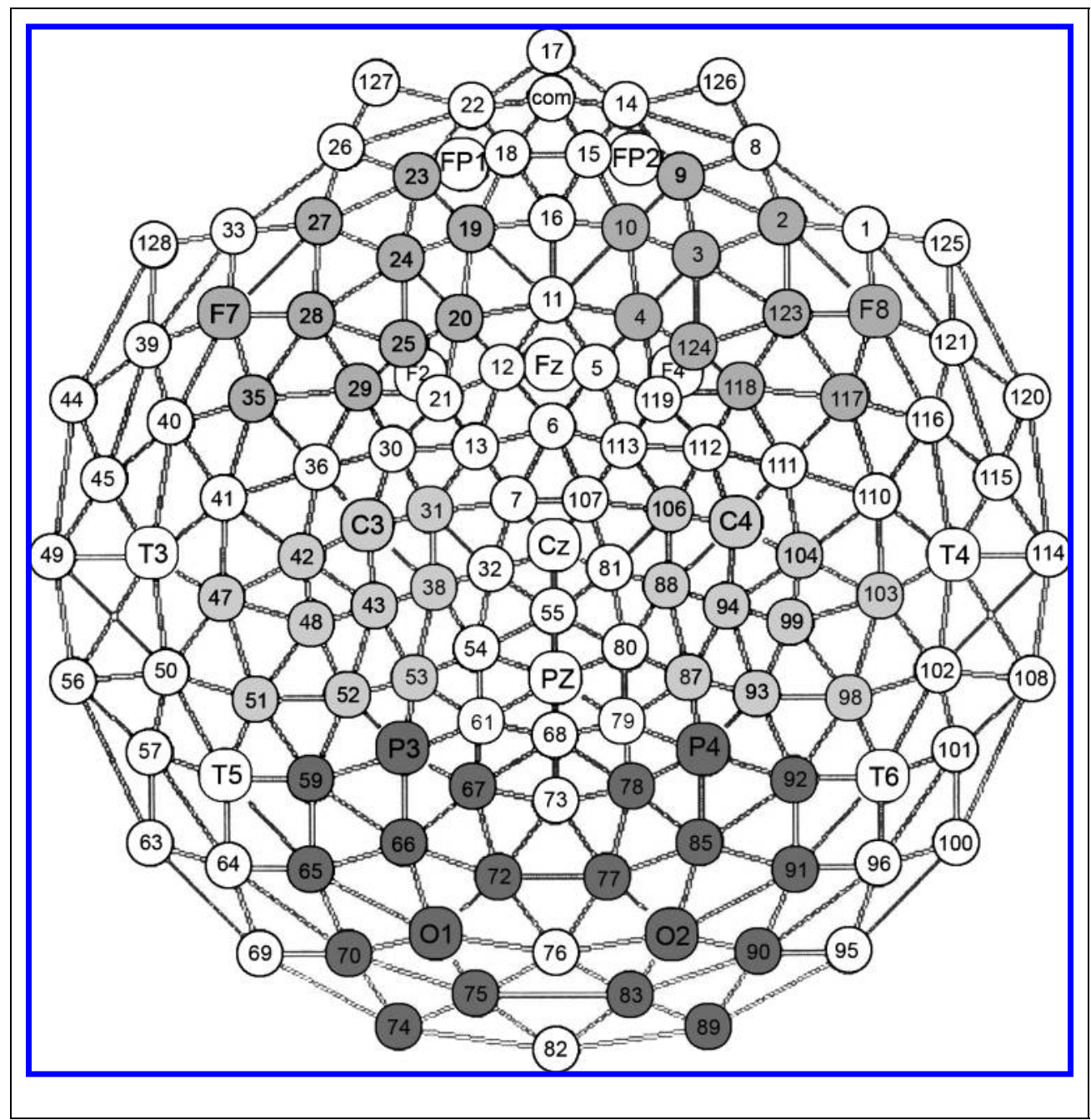


Baseline correction was performed using the average EEG activity in the $100 \mathrm{msec}$ preceding the onset of the target word as a reference signal value. Following baseline correction, epochs with simultaneous artifacts in at least 10 channels were rejected. In addition, trials that were not responded to correctly were not included in the analysis. This resulted in the exclusion of approximately $5 \%$ of the trials, which were evenly distributed among the different experimental conditions. Furthermore, electrodes with a high level of rejected trials $(>10 \%)$ were substituted by the average value of the group of nearest electrodes. Separate ERPs were formed for each of the experimental conditions, each of the subjects, and each of the electrode sites.

Six regions of interest were computed out of the 129 electrodes, each containing the mean of a group of electrodes. The regions were as follows (see electrode numbers in Figure 6): left anterior (19, 20, 23, 24, 25, 27, 28, 29, 34, 35), left central $(31,37,38,42,43,47,48,51$, $52,53)$, left posterior $(59,60,65,66,67,70,71,72,74$, 75), right anterior $(2,3,4,9,10,117,118,122,123,124)$, right central $(87,88,93,94,98,99,103,104,105,106)$, right posterior $(77,78,83,84,85,86,89,90,91,92)$.

Mean amplitudes were obtained for different time windows. For each measure a repeated measures ANOVA was performed, including electrode regions (anterior, central, and posterior), hemisphere (left/right), and the experimental variables as additional factors. These variables were color-syllable congruency (congruent, incongruent, and baseline) and lexicality (words vs. pseudowords) in Experiment 1, and color-syllable congruency (congruent vs. incongruent) and lexicality (word vs. pseudoword) or lexical frequency (high and low) in Experiment 2. Where appropriate, critical values were adjusted using the Greenhouse and Geisser (1959) correction for violation of the assumption of sphericity. Effects for the electrode region factor or for the hemisphere factor were reported only when they interacted with the experimental manipulations.

\section{Stimuli}

\section{Experiment 1}

One hundred twenty bisyllabic words with CV.CV ${ }^{4}$ and CV.CVC structures were selected from the Spanish word pool LEXESP (Sebastián-Gallés, Martí, Carreiras, \& Cuetos, 2000). The mean frequency was 8.7 per million (range, 3-18). Thus, all words can be considered as low frequency. The frequency of the first syllable as well as the bigram frequency was controlled (bigram frequency intrasyllable was always less or equal to the bigram frequency of the trough between the first and the second syllables). In addition, 120 pseudowords, half of them with a CV.CV structure and the other half with a CV.CVC structure, were created by changing one letter from existing words in Spanish and preserving the phonotactic and orthotactic rules of Spanish. In addition, we selected another 120 words and created another 120 pseudowords, all of similar length (five and six letters long) but with different initial syllables (e.g., CVC and CCV initial syllables) to divert the attention of participants from the critical syllable structure. See Table 4 for a summary of the different characteristics of the materials.

The items (words and pseudowords) were colored with either the whole word in green or red (baseline conditions) or part of the word in green and the other part in red (congruent and incongruent conditions). In the congruent condition, there was congruency between the boundaries of the two syllables of the word and the boundaries of the two colors, whereas in the incongruent condition there was none. Thus, for instance, Figure 5 shows the word "casino" colored as it appeared in the incongruent condition. In the congruent condition, the first syllable "ca" was colored in one of the colors and the rest of the word "sino" in the other color. Three versions of color combinations (congruent, incongruent, and baseline) were created for each item and the order of colors (red-green vs. green-red) was counterbalanced. The proportion of green and red letters was the same for congruent and incongruent trials. That is, the same number of letters was colored in red and green across trials. Six lists of 480 items were created so that each item appeared in all six counterbalanced conditions across the lists, but only in one condition within each list. Because each subject was assigned only to one list, the participant saw only one version of each item.

\section{Experiment 2}

One hundred sixty bisyllabic and trisyllabic words with CV.CV and CV.CV.CV structures, respectively, were selected from the Spanish word pool LEXESP (SebastiánGallés et al., 2000). Eighty words were of low printed

Table 4. Syllable and Bigram Type Frequency of Stimuli of Experiments 1 and 2

\begin{tabular}{lcccc}
\hline & Syllable 1 & Syllable 2 & $\begin{array}{c}\text { Bigram } \\
\text { Intra }\end{array}$ & $\begin{array}{c}\text { Bigram } \\
\text { Inter }\end{array}$ \\
\hline $\begin{array}{l}\text { Experiment 1 } \\
\text { Words }\end{array}$ & 16.4 & 11.1 & 22.1 & 39.6 \\
Pseudowords & 14.3 & 6.5 & 15.1 & 20.2 \\
& & & & \\
Experiment 2 & & & & \\
HF Words & 49.3 & 44.3 & 57.6 & 59.3 \\
LF Words & 33.5 & 44.9 & 48.4 & 54.2 \\
Pseudowords & 39.2 & 39.4 & 40.3 & 43.9 \\
\hline
\end{tabular}


frequency and the other 80 were of high frequency. The mean frequency of the low-frequency group was 4.3 (range, 2-7), whereas the mean frequency of the highfrequency group was 57.8 (range, 19-206). The frequency of the first syllable as well as the bigram frequency was controlled: The bigram frequency intrasyllable was always less or equal to the bigram frequency of the trough between the first and the second syllables. In addition, another 160 pseudowords, half of them with a CV.CV structure and the other half with a CV.CV.CV structure were created by changing one letter from existing words in Spanish and preserving the phonotactic and orthotactic rules of Spanish. Finally, we selected another 240 words and created another 240 pseudowords, all of similar length (four, five, and six letters long) but with different initial syllables (e.g., CVC and CCV initial syllables) to divert the attention of participants from the critical syllable structure. See Table 4 for a summary of the different characteristics of the materials.

In Experiment 2 only congruent and incongruent conditions were produced. Thus, items always appeared in two colors-red and green-so that in the congruent condition the first syllable was in one color and the rest of the word in the other color, that is, the color boundaries matched the limit of the first syllable, whereas in the incongruent condition color boundaries and first syllable did not match. Two versions of color combinations (congruent vs. incongruent with syllable boundaries) were created for each item and the order of colors (red-green vs. green-red) was counterbalanced. Four lists of 800 items were created so that each item appeared in all four counterbalanced conditions across lists, but only in one condition within each list. Because each subject was assigned to only one list he or she saw only one version of each item.

\section{Acknowledgments}

This work was supported by grants BSO2001-3492-C04-03 and BS02003-01135 (Spanish Ministry of Science and Technology). We thank Peter Hagoort, Phillip Holcomb, Marta Kutas, Thomas Muente, and two anonymous reviewers for comments on previous versions of the manuscript.

Reprint requests should be sent to Manuel Carreiras, Departamento de Psicología Cognitiva, Campus de Guajara, Universidad de La Laguna, Tenerife 38205, Spain, or via e-mail: mcarreir@ull.es). URL: www.neurocog.ull.es.

\section{Notes}

1. Periods signal the syllable boundaries and were not displayed in the original experimental stimuli.

2. The words baza, bedel, fotón, and mole were excluded from the analyses because more than $50 \%$ of subjects misclassified them as pseudowords.

3. The words tufo, bazo, and pecata were excluded from the analyses because more than $50 \%$ of subjects misclassified them as pseudowords.

4. C stands for consonant and V for vowel.

\section{REFERENCES}

Álvarez, C. J., Carreiras, M., \& Perea, M. (2004). Are syllables phonological units in visual word recognition? Language and Cognitive Processes, 19, 427-452.

Álvarez, C. J., Carreiras, M., \& Taft, M. (2001). Syllables and morphemes: Contrasting frequency effects in Spanish. Journal of Experimental Psychology: Learning, Memory, and Cognition, 27, 545-555.

Andrews, S. (1997). The effects of orthographic similarity on lexical retrieval: Resolving neighborhood conflicts. Psychological Bulletin and Review, 4, 439-461.

Ans, B., Carbonnel, S., \& Valdois, S. (1998). A connectionist multiple-trace model for polysyllabic word reading. Psychological Review, 105, 678-723.

Barber, H., Vergara, M., \& Carreiras, M. (2004). Syllable-frequency effects in visual word recognition: Evidence from ERPs. NeuroReport, 15, 545-548.

Bentin, S., McCarthy, G., \& Wood, C. (1985). Event-related potentials, lexical decision and semantic priming. Electroencephalography and Clinical Neurophysiology, 60, 343-355.

Bentin, S., Mouchetant-Rostaing, Y., Giard, M. H., Echallier, J. F., \& Pernier, J. (1999). ERP manifestations of processing printed words at different psycholinguistic levels: Time course and scalp distribution. Journal of Cognitive Neuroscience, 11, 35-60.

Carreiras, M., Álvarez, C. J., \& de Vega, M. (1993). Syllable frequency and visual word recognition in Spanish. Journal of Memory and Language, 32, 766-780.

Carreiras, M., \& Grainger, J. (2004). Sublexical representations and the "front end" of visual word recognition. Language and Cognitive Processes, 19, 321-331.

Carreiras, M., \& Perea, M. (2002). Masked priming effects with syllabic neighbors in a lexical decision task. Journal of Experimental Psychology: Human Perception and Performance, 28, 1228-1242.

Coltheart, M., Rastle, K., Perry, C., Langdon, R., \& Ziegler, J. (2001). DRC: A dual route cascaded model of visual word recognition and reading aloud. Psychological Review, 108, 204-256.

Conrad, M., \& Jacobs, A. (2004). Replicating syllable frequency effects in Spanish in German: One more challenge to computational models of visual word recognition. Language and Cognitive Processes, 19, 369-390.

Ferrand, L., Segui, J., \& Grainger, J. (1996). Masked priming of words and picture naming: The role of syllabic units. Journal of Memory and Language, 35, 708-723.

Grainger, J., \& Jacobs, A. M. (1996). Orthographic processing in visual word recognition: A multiple read-out model. Psychological Review, 22, 696-713.

Greenhouse, S., \& Geisser, S. (1959). On methods in the analysis of profile data. Psychometrika, 24, 95-112.

Hagoort, P. (2003). Interplay between syntax and semantics during sentence comprehension: ERP effects of combining syntactic and semantic violations. Journal of Cognitive Neuroscience, 15, 883-899.

Holcomb, P. J., Grainger, J., \& O'Rourke, T. (2002). An Electrophysiological study of the effects of orthographic neighborhood size on printed word perception. Journal of Cognitive Neuroscience, 14, 938-950.

King, J. W., \& Kutas, M. (1998). Neural plasticity in the dynamics of human visual word recognition. Neuroscience Letters, 1-4.

Kutas, M., \& Federmeier, K. D. (2000). Electrophysiology reveals semantic memory use in language comprehension. Trends in Cognitive Sciences, 4, 463-470. 
Mathey, S., \& Zagar, D. (2002). Lexical similarity in visual word recognition: The effect of syllabic neighborhood in French. Current Psychology Letters, 8, 107-121.

McClelland, J. L., \& Rumelhart, D. E. (1981). An interactive activation model of context effects in letter perception: Part 1. An account of basic findings. Psychological Review, 88, 375-407.

Münte, T. F., Wieringa, B. M., Weyerts, H., Szentkuti, A., Matzke, M., \& Johannes, S. (2001). Differences in brain potentials to open and closed class words: Class and frequency effects. Neuropsychologia, 39, 91-102.

Neville, H. J., Mills, D. L., \& Lawson, D. S. (1992). Fractionating language: Different neural subsystems with different sensitive periods. Cerebral Cortex, 2, 244-258.

Oldfield, R. C. (1971). The assessment and analysis of handedness. The Edinburgh Inventory. Neuropsychologia, 9, 97-113.

Osterhout, L., Bersick, M., \& McKinnon, R. (1997). Brain potentials elicited by words: Word length and frequency predict the latency of an early negativity. Biological Psychology, 46, 143-168.

Perea, M., \& Carreiras, M. (1998). Effects of syllable frequency and syllable neighborhood frequency in visual word recognition. Journal of Experimental Psychology: Human Perception and Performance, 24, 134-144.

Plaut, D. C., McClelland, J. L., Seidenberg, M. S., \& Patterson, K. (1996). Understanding normal and impaired word reading: Computational principles. Psychological Review, 103, 56-115.

Rayner, K. (1975). The perceptual span and peripheral cues in reading. Cognitive Psychology, 7, 65-81.

Rayner, K. (1998). Eye movements in reading and information processing: 20 years of research. Psychological Bulletin, 124, 372-422.

Rugg, M. D. (1984a). Event-related potentials in phonological matching tasks. Brain and Language, 23, 225-240.

Rugg, M. D. (1984b). Event-related potentials and the phonological processing of words and non-words. Neuropsychologia, 22, 435-443.
Schendan, H. E., Giorgio, G., \& Kutas, M. (1998). Neurophysiological evidence for visual perceptual categorization of words and faces within $150 \mathrm{~ms}$. Psychophysiology, 35, 240-251.

Sebastián-Gallés, N., Martí, M. A., Carreiras, M., \& Cuetos, F. (2000). Lexesp: Una base de datos informatizada del español. Barcelona, Spain: Universitat De Barcelona.

Seidenberg, M. S., \& McClelland, J. L. (1989). A distributed, developmental model of word recognition and naming. Psychological Review, 96, 523-568.

Sereno, S., \& Rayner, K. (2003). Measuring word recognition in reading: Eye movements and event-related potentials. Trends in Cognitive Sciences, 7, 489-493.

Sereno, S. C., Rayner, K., \& Posner, M. I. (1998). Establishing a time-line of word recognition: Evidence from eye movements and event-related potentials. NeuroReport, 9, 2195-2200.

Starr, M. S., \& Rayner, K. (2001). Eye movements during reading: Some current controversies. Trends in Cognitive Sciences, 5, 156-163.

Taft, M. (1979). Lexical access via an orthographic code: The BOSS. Journal of Verbal Learning and Verbal Behavior, 18, 21-39.

Taft, M., \& Forster, K. I. (1976). Lexical storage and retrieval of polymorphemic and polysyllabic words. Journal of Verbal Learning and Verbal Behavior, 15, 607-620.

ter Keurs, M., Brown, C. M., \& Hagoort, P. (2002). Lexical processing of vocabulary class in patients with Broca's aphasia: An event-related brain potential study on agrammatic comprehension. Neuropsychologia, 40, 1547-1561.

ter Keurs, M., Brown, C. M., Hagoort, P., \& Stegeman, D. F. (1999). Electrophysiological manifestations of open- and closed-class words in patients with Broca's aphasia with agrammatic comprehension. An event-related brain potential study. Brain, 122, 839-854.

Van Petten, C., \& Kutas, M. (1990). Interactions between sentence context and word frequency in event-related brain potentials. Memory and Cognition, 18, 380-393. 


\section{This article has been cited by:}

1. M. Carreiras, J. A. Dunabeitia, N. Molinaro. 2009. Consonants and Vowels Contribute Differently to Visual Word Recognition: ERPs of Relative Position Priming. Cerebral Cortex 19:11, 2659-2670. [CrossRef]

2. Manuel Carreiras, Jordi Riba, Marta Vergara, Marcus Heldmann, Thomas F. Münte. 2009. Syllable congruency and word frequency effects on brain activation. Human Brain Mapping 30:9, 3079-3088. [CrossRef]

3. FABIENNE CHETAIL, STEPHANIE MATHEY. 2009. Activation of syllable units during visual recognition of French words in Grade 2. Journal of Child Language 36:04, 883. [CrossRef]

4. Manuel Carreiras, Manuel Perea, Marta Vergara, Alexander Pollatsek. 2009. The time course of orthography and phonology: ERP correlates of masked priming effects in Spanish. Psychophysiology 46:5, 1113-1122. [CrossRef]

5. Manuel Carreiras, Margaret Gillon-Dowens, Marta Vergara, Manuel Perea. 2009. Are Vowels and Consonants Processed Differently? Event-related Potential Evidence with a Delayed Letter ParadigmAre Vowels and Consonants Processed Differently? Event-related Potential Evidence with a Delayed Letter Paradigm. Journal of Cognitive Neuroscience 21:2, 275-288. [Abstract] [Full Text] [PDF] [PDF Plus]

6. Manuel Carreiras, Marta Vergara, Manuel Perea. 2009. ERP correlates of transposed-letter priming effects: The role of vowels versus consonants. Psychophysiology 46:1, 34-42. [CrossRef]

7. Ruth Filik, Anthony J. Sanford, Hartmut Leuthold. 2008. Processing Pronouns without Antecedents: Evidence from Event-related Brain PotentialsProcessing Pronouns without Antecedents: Evidence from Event-related Brain Potentials. Journal of Cognitive Neurosience 20:7, 1315-1326. [Abstract] [PDF] [PDF Plus]

8. Chia-Ying Lee, Jie-Li Tsai, Wen-Hsuan Chan, Chun-Hsien Hsu, Daisy L. Hung, Ovid J.L. Tzeng. 2007. Temporal dynamics of the consistency effect in reading Chinese: an event-related potentials study. NeuroReport 18:2, 147-151. [CrossRef] 